\title{
Uveíte anterior na ausência de esclerite em paciente com artrite reumatóide: relato de caso
}

\author{
Anterior uveitis in the absence of scleritis in a pacient with rheumathoid arthritis: case report
}

Carolina Rottill Daguano ${ }^{1}$, Claudia Regina Bochnia ${ }^{1}$, Marcelo Gehlen²

\section{RESUMO}

A artrite reumatóide é a colagenose mais comum, afetando cerca de 0,6\% da população brasileira e é uma grande causadora de deformidades articulares em mais variadas formas. A principal manifestação ocular da artrite reumatóide é a ceratoconjuntivite sicca (Sjögren secundária), seguida pela esclerite, úlcera periférica da córnea e uveíte. O objetivo deste trabalho é apresentar um caso de uveíte anterior em paciente com artrite reumatóide, uma apresentação rara em pacientes com esta patologia. Paciente feminina, 55 anos, portadora de artrite reumatóide, apresentando quadro de dor e piora súbita da acuidade visual no olho direito. O exame mostrava reação de câmara anterior com hipópio, úlcera corneana periférica e pressão intraocular de $32 \mathrm{mmHg}$. Foi realizado o diagnóstico de uveíte anterior hipertensiva e úlcera corneana periférica e realizado tratamento com corticóide oral e tópico, antibiótico tópico, colírio cicloplégico e hipotensores oculares tópicos e sistêmicos. Os casos de uveíte anterior são comuns em doenças reumatológicas, principalmente em artropatias soronegativas relacionadas ao HLA-B27, conferindo grande causa de morbidade a esses pacientes. Neste trabalho relatamos um caso de uveíte anterior em paciente com artrite reumatóide, uma apresentação rara encontrada na literatura médica atual.

Descritores: Artrite reumatóide/complicações; Ceratoconjuntivite seca/etiologia; Esclerite; Úlcera da córnea; Uveíte anterior/quimioterapia; Corticosteróides/uso terapêutico; Antígeno HLA-DR4; Antígeno HLA-B27; Humanos; Meia-idade; Feminino; Relatos de casos

\begin{abstract}
Rheumathoid arthritis is the most common collagenosis and affects almost $0.6 \%$ of brazilian population. It is an important cause of articular deformities. The main ocular manifestation of rheumathoid arthritis is dry eyes (secondary Sjögren's syndrome), followed by scleritis, peripheral ulcerative keratitis and uveitis. The aim of this paper is to present a case of anterior uveitis in the absence of scleritis in a patient with rheumathoid arthritis, a very rare presentation in this type of patient. Female patient, 55 years old, with rheumathoid arthritis, presenting suddenly ocular pain and low vision in the right eye. Her exam showed anterior chamber reaction with hypopion, peripheral corneal keratitis and intraocular pressure of $32 \mathrm{mmHg}$. She was diagnosed with hypertensive anterior uveitis and peripheral corneal keratitis and treated with systemic and topical corticosteroids, topical antibiotic, topic and systemic ocular hypotensive and mydriatic drops. Anterior uveitis is common in rheumatological diseases, especially in those soronegative arthropathies related to HLA B27. In this paper we present a patient with rheumathoid arthritis and anterior uveitis in the absence of scleritis, a rare presentation in actual medical literature.
\end{abstract}

Keywords: Arthritis, rheumatoid/complications; Keratoconjunctivitis sicca/etiology; Scleritis; Corneal ulcer; Uveitis, anterior/drug therapy: Adrenal cortex hormones/therapeutic use; HLA-DR4 antigen; HLA-B27 antigen; Humans; Middle aged; Female; Case reports

\section{INTRODUÇÃO}

A artrite reumatóide (AR) é a colagenose mais comum, afetando cerca de 0,6\% da população brasileira e é uma grande causadora de deformidades articulares em mais variadas formas ${ }^{(1)}$.

Antigamente considerada uma doença crônica e benigna, atualmente se sabe que a AR tem um potencial destrutivo, levando à incapacidade 10\% dos pacientes após 10 anos de doença e 90\% deles após 30 anos. O portador de AR tem sua sobrevida comprovadamente diminuída e a principal causa de mortalidade nesses pacientes é a doença cardiovascular(2). Segundo alguns estudos, uma pessoa com AR, com envolvimento de 30 ou mais articulações, tem um prognóstico equivalente ao de uma doença coronoriana de três vasos ou linfoma Hodgkin em estágio avançado ${ }^{(1,3)}$.

A artrite reumatóide afeta predominantemente mulheres (3:1) na meia-idade e está associada à presença de HLA-DR4 e -DR1 positivos. A apresentação clássica é a de uma poliartrite crônica aditiva que afeta todas as articulações sinoviais, predominantemente as pequenas. Algumas são poupadas como as interfalangianas distais e as da coluna torácica e lombar ${ }^{(1)}$.
Existem várias manifestações extra-articulares, a mais comum é a presença de nódulos reumatóides que aparecem em pontos de atrito como a superfície flexora dos cotovelos, do tendão de Aquiles, etc. Também ocorrem manifestações pulmonares (síndrome de Caplan), cardíacas (pericardites, miocardites), neurológicas (polineuropatias, mononeurite multiplex), musculares (miosites, atrofia por desuso), cutâneas (vasculites, úlceras, fenômeno de Raynaud), hematológicas (anemia, leucopenia: síndrome de Felty) e oculares (episclerite, esclerite, escleromalácia perfurante, Sjögren secundário e uveíte) $)^{(1)}$.

As principais manifestações oculares da AR são a ceratoconjuntivite sicca (Sjögren secundário), seguida pela esclerite, úlcera periférica da córnea e episclerite. A uveíte é uma manifestação ocular incomum nesses pacientes ${ }^{(4-5)}$.

A uveíte anterior é a apresentação mais comum das seguintes doenças reumáticas: artrite idiopática juvenil, espondilite anquilosante, síndrome de Reiter e artrite psoriática ${ }^{(4-5)}$.

Neste relato de caso, apresenta-se uma paciente com artrite reumatóide com quadro de uveíte anterior na ausência de escleri- 
te. De acordo com a literatura, esta é uma manifestação ocular não usual e rara, sendo por isso, relatada neste trabalho.

\section{RELATO DO CASO}

Paciente feminina, 55 anos, sexo feminino, branca, brasileira, residente em Curitiba, com história de turvação no olho direito (OD) há três meses, procura a emergência de oftalmologia por hiperemia e dor no olho direito há cinco dias, além de piora súbita da acuidade visual. Negava secreção, prurido ou sensação de corpo estranho. A paciente relatava ser portadora de artrite reumatóide há 25 anos e estava em acompanhamento no serviço de Reumatologia do Hospital de Clínicas - UFPR.

Ao exame oftalmológico, apresentava acuidade visual de 20/100 no OD e 20/25 no olho esquerdo (OE). A biomicroscopia do OD apresentava hiperemia perilimbar intensa, ausência de sinais de esclerite, câmara anterior rasa com íris bombé, reação de câmara anterior com flare $++/ 4+$ e células $+++/ 4+$, hipópio de 2 mm, seclusão pupilar, corectopia e ceratite marginal de $2 \mathrm{~mm}$ às 10 horas com afinamento corneano. A medida da pressão intraocular (PIO) com tonômetro de Goldmann era $32 \mathrm{mmHg}$ e o fundo de olho era impraticável nesse olho. A biomicroscopia do OE mostrava uma câmara anterior profunda e sem alterações e a medida da PIO no OE era $12 \mathrm{mmHg}$. Ao exame clínico, a paciente apresentava deformidades das articulações interfalangianas proximais e médias, com leve edema e hiperemia.

Foi realizado tratamento de emergência para o aumento da PIO com manitol $250 \mathrm{ml}$ EV e acetazolamida $250 \mathrm{mg} 2$ comprimidos via oral, o qual apresentou bom resultado, reduzindo a PIO para $16 \mathrm{mmHg}$. Após o tratamento inicial, foram iniciados ciprofloxacino + dexametasona colírio 6 vezes ao dia, maleato de timolol 0,5\% colírio 2 vezes ao dia, cicloplégico colírio 2 vezes ao dia e lubrificante ocular várias vezes ao dia. Sistemicamente, foram prescritos prednisona $1 \mathrm{mg} / \mathrm{kg}$ peso corporal via oral ao dia e acetazolamida $250 \mathrm{mg}$ via oral 2 vezes ao dia.

Após duas semanas de tratamento, a paciente apresentava piora da acuidade visual no OD, apenas contando dedos a 2 metros. Mantinha a reação na câmara anterior com hipópio menor do que na primeira consulta ( $1 \mathrm{~mm}$ ) e a ceratite marginal de $2 \mathrm{~mm}$ às $10 \mathrm{~h}$, apresentando também depósitos de fibrina na câmara anterior e no endotélio corneano os quais justificaram a piora da acuidade visual (Figura 1). Após 2 dias, apresentou acuidade visual de 20/100, diminuição dos depósitos corneanos e na câmara anterior e PIO de $10 \mathrm{mmHg}$ no OD, sem demais alterações na biomicroscopia, sendo então realizada uma injeção subconjuntival de 0,7 ml de dexametasona.

Durante o acompanhamento ambulatorial, foi realizada a pesquisa do FAN, fator reumatóide e HLA-B27, os quais foram todos não reagentes. Analisando seu acompanhamento reumatológico, a paciente já possuía um diagnóstico de artrite reumatóide com fator reumatóide negativo confirmado pela Reumatologia.

Nas avaliações subsequentes, houve uma melhora importante da acuidade visual, chegando a 20/30 com a melhor correção e a PIO manteve-se controlada. Ao exame final, a paciente apresentava o olho calmo com corectopia, seclusão pupilar, catarata subcapsular posterior e nuclear, leucoma corneano marginal e ausência de reação na câmara anterior.

\section{DISCUSSÃO}

A uveíte anterior é a mais comum das uveítes, sendo frequentemente associada a processos sistêmicos inflamatórios e infecciosos. É uma doença aguda, recorrente e que leva à perda visual grave ou cegueira em 20\% dos $\operatorname{casos}^{(4)}$.

Dentre as doenças inflamatórias, é mais comumente relacionada com o espectro de doenças HLA-B27 positivas, sendo a espondiloartropatia detectada em $65 \%$ dos pacientes com doença reumatológica(4-5).

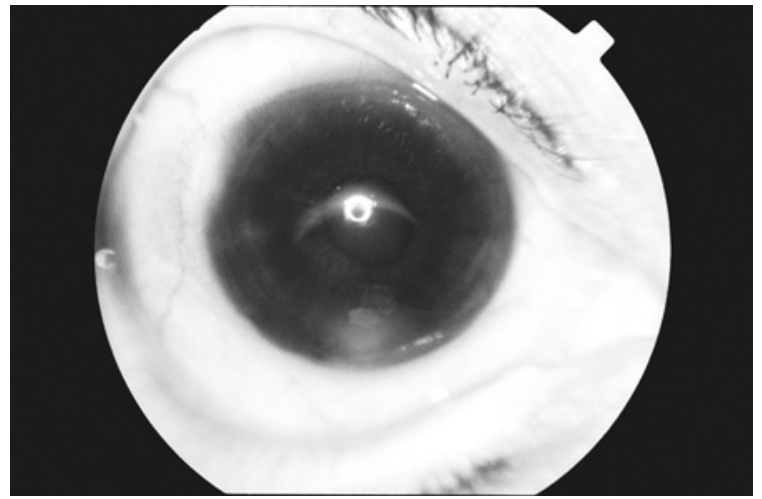

Figura 1. Biomiscroscopia mostrando fibrina inferior na câmara anterior, precipitados ceráticos e corectopia.

A etiologia da artrite reumatóide (AR) permanece desconhecida, mas muito já se sabe sobre sua imunopatologia. A doença é o resultado da interação entre genética, ambiente (gatilho infeccioso?) e desequilíbrio do sistema imunológico(6).

Apesar de as maiores manifestações se relacionarem ao sistema musculoesquelético, a AR pode afetar qualquer órgão ou tecido. A manifestação ocular mais comum na artrite reumatóide é o olho seco decorrente da síndrome de Sjogren secundária, o qual causa muitos sintomas desconfortáveis, mas raramente leva à perda visual e geralmente responde bem ao tratamento clínico com lubrificantes oculares ${ }^{(5)}$.

A esclerite anterior está relacionada a doenças reumatológicas em cerca de 30 a 40\% dos casos, sendo a artrite reumatóide a associação mais comum ${ }^{(7)}$. Geralmente se apresenta em pacientes com doença avançada e outras manifestações extra-articulares. Caracteriza-se por uma vasculite granulomatosa e sua resposta ao tratamento tópico é pobre ${ }^{(5)}$.

A ceratite geralmente ocorre no curso de uma esclerite, porém pode ocorrer isoladamente como uma úlcera periférica inflamatória ou afinamento corneano central ou periférico em olhos não inflamados. No caso presente, foi observada úlcera periférica não associada à esclerite anterior, a qual não demonstrou comportamento agressivo e teve boa resposta ao uso de corticoesteróides tópicos e lubrificantes oculares.

A uveíte anterior não é uma manifestação extraocular comum da artrite reumatóide. Este caso clínico é um relato raro na literatura, mostrando uma paciente com diagnóstico de artrite reumatóide avançada e uveíte anterior na ausência de esclerite.

Estudos mostram que cerca de $60 \%$ dos pacientes com uveíte anterior possuem doença reumatológica, enquanto cerca de $40 \%$ permanecem sem diagnóstico de doença sistêmica. Entretanto, mesmo aqueles pacientes sem doença de base associada respondem bem à terapia com as drogas antirreumáticas modificadoras da doença (DMARDs) $)^{(4)}$.

\section{REFERÊNCIAS}

1. Gehlen M, Skare TL. Reumato-oftalmologia. São Paulo: Editora Tecmed; 2007.

2. Pereira IA, Borba EF. Multiple factors determine the increased prevalence of atherosclerosis in rheumatoid arthritis. Acta Reumatol Port. 2008;33(1):47-55.

3. Sokka T, Abelson B, Pincus T. Mortality in rheumatoid arthritis: 2008 update. Clin Exp Rheumatol. 2008;26(5 Suppl 51):S35-61.

4. Bachta A, Tlustocowicz M, Staniszewska J. Idiopathic anterior uveitis: is it a rheumatic disease? Joint Bone Spine. 2007;74(3):215-21.

5. McCluskey P, Powell RJ. The eye in systemic inflammatory diseases. Lancet. 2004;364(9451): 2125-33.

6. Sturrock RD. Update on the pathogenesis of rheumatoid arthritis. International Congress Series. 2006;1295:1-8.

7. Akpek EK, Thorne JE, Qazi FA, Do DV, Jabs DA. Evaluation of patients with scleritis for systemic disease. Ophthalmology. 2004;111(3):501-6. Comment in: Ophthalmology. 2007; 114(6):1232. 